\title{
La incapacidad temporal como beneficio de la seguridad social: una revisión de literatura ${ }^{1}$
}

\author{
Sick leave as a social security benefit: A literature review \\ Licença médica como benefício da previdência social: \\ uma revisão de literatura
}

\author{
Jenny Andrea Beltrán Torres ${ }^{2}$ \\ Ivonne Constanza Valero-Pacheco ${ }^{3}$ \\ Miguel Antonio Hernández Rodiño ${ }^{4}$ \\ Luisa Fernanda Penagos Simbaqueba ${ }^{5}$ \\ Andrés Felipe Perdomo Riveros ${ }^{6}$
}

Recibido: 22 de diciembre 2020 • Enviado para modificación: 23 de marzo 2021 • Aceptado: 26 de abril 2021

Beltrán Torres, J. A., Valero-Pacheco, I. C., Hernández Rodiño, M. A., Penagos Simbaqueba, L. F. y Perdomo Riveros, A. F. (2021). La incapacidad temporal como beneficio de la seguridad social: una revisión de literatura. Revista Ocupación Humana, 21(1), 42-57. https://doi.org/10.25214/25907816.1032

\section{RESUMEN}

La incapacidad temporal es el beneficio asistencial y económico que se le reconoce a trabajadoras y trabajadores cotizantes del sistema de seguridad social por la afectación en su

\footnotetext{
${ }^{1}$ Este artículo es resultado parcial del proyecto Las Incapacidades Temporales como Factor de Ineficiencia y Desperdicio en el Sistema de Salud Colombiano - Fase 1 dirigido por la profesora Ivonne Constanza Valero-Pacheco. Proyecto aprobado en la Convocatoria Interna de Investigación n. ${ }^{\circ} 17$ de 2018, con la Referencia 921-17-18, por la Dirección de Investigación, Creatividad e Innovación de la Universidad de Bogotá Jorge Tadeo Lozano, y como parte del Grupo de Investigación en Gestión de Salud, Trabajo y en Políticas Sociales.

${ }^{2}$ Fisioterapeuta. Especialista en Gestión de la Seguridad y Salud en el Trabajo. Sanatorio Agua de Dios. Agua de Dios, Colombia. jennya.beltrant@utadeo.edu.co. iD https://orcid.org/0000-0001-5087-3721

${ }^{3}$ Terapeuta ocupacional. Abogada. Especialista en Administración de Salud Ocupacional. Magíster en Docencia e Investigación Universitaria. Profesora asociada, Universidad de Bogotá Jorge Tadeo Lozano. Bogotá, Colombia. ivonne.valero@utadeo.edu.co iD https://orcid.org/0000-0002-3217-0800

${ }^{4}$ Ingeniero ambiental. Especialista en Gestión de la Seguridad y Salud en el Trabajo. Clínica Materno Infantil Casa del Niño. Montería, Colombia. miguel.hernandezr@utadeo.edu.co iD https://orcid.org/0000$\underline{0003-1494-2261}$

${ }^{5}$ Enfermera. Especialista en Gestión de la Seguridad y Salud en el Trabajo. Hospital Universitario Mayor Méderi. Bogotá, Colombia. fernanda.penagoss@utadeo.edu.co iD https://orcid.org/0000-0003-4188-2233

${ }^{6}$ Ingeniero industrial. Especialista en Gestión de la Seguridad y Salud en el Trabajo. Independiente. Bogotá, Colombia. andresf.perdomor@utadeo.edu.co iD https://orcid.org/0000-0001-7610-1180
} 
capacidad laboral e implica ausentismo y asistencia para el retorno al trabajo. El objetivo de esta revisión es explorar las concepciones, los sujetos y las situaciones protegidas por esta prestación en diferentes países. Se realizó una revisión de literatura en bases de datos y sitios web oficiales de Colombia, Argentina, Chile, Perú, Ecuador, México, España y Portugal. Para el análisis se diseñaron matrices y un cuadro descriptivo con identificadores normativos; los hallazgos se organizaron en categorías. Se encontraron semejanzas en los elementos conceptuales, eventos y sujetos de protección. Las diferencias están en los requisitos y duración. La incapacidad temporal es un beneficio universal y finito reconocido como un derecho laboral y de la seguridad social. Por su impacto económico y social, debe ser estudiada desde la gestión en salud y de los riesgos laborales para el diseño de planes y programas, así como de estrategias regionales con enfoque de equidad, considerando la ocupación y el ámbito laboral.

\title{
PALABRAS CLAVE
}

ausencia por enfermedad, incapacidad laboral transitoria, beneficios del seguro, seguridad social, absentismo

\begin{abstract}
Sick leave is an economic and support benefit of a social security system for workers who contribute to it, when their work capacity has been impacted. It implies absenteeism and assistance to return to work. This review aims to explore the conceptions and protected subjects and events of this benefit in different countries. A literature review was carried out on databases and official websites of Colombia, Argentina, Chile, Perú, Ecuador, México, Spain, and Portugal. For the analysis, matrices and a descriptive table with normative identifiers were designed. The findings were organized into categories. Similarities were found in conceptual elements and the protected events and subjects. The differences include the requirements and duration. Sick leave is a universal and finite benefit recognized as a labor and social security right. Due to its economic and social impact, it must be studied from health and occupational risks management to design plans and programs and regional strategies focusing on equity, considering the occupation and the work environment.
\end{abstract}

\section{KEY WORDS}

sick leave, insurance benefits, social security, absenteeism

\section{RESUMO}

Licença médica é o benefício assistencial e econômico que é reconhecido aos trabalhadores e trabalhadoras contribuintes do sistema de previdência social pelo impacto na sua capacidade para o trabalho e implica absentismo e assistência no regresso ao trabalho. O objetivo desta revisão é explorar as concepções, temas e situações protegidas por esse benefício em diferentes países. Foi realizada uma revisão da literatura em bases de dados e em sites oficiais da Colômbia, Argentina, Chile, Peru, Equador, México, Espanha e Portugal. Para a análise, foram elaboradas matrizes e uma tabela descritiva com identificadores normativos; os resultados foram organizados em categorias. Semelhanças foram encontradas nos elementos conceituais, eventos e temas de proteção. As diferenças estão nos requisitos e na duração. A licença médica é um benefício universal e finito, reconhecido como direito trabalhista e previdenciário. Devido ao seu impacto econômico e social, deve ser estudada 
a partir da gestão em saúde e dos riscos trabalhistas para o esboço de planos e programas; bem como das estratégias regionais com foco na equidade, considerando a ocupação e o ambiente de trabalho.

\section{PALAVRAS-CHAVE}

licença médica, benefícios de seguro, previdência social, absenteísmo

\section{Introducción}

La incapacidad temporal es un beneficio de la seguridad social que provee la asistencia para la recuperación y compensa la falta de ingresos de la o el trabajador (Valero-Pacheco, 2020). Para las organizaciones, es una clase de absentismo justificado del trabajador o trabajadora (Antonio, 2011; Álvarez, 2017) debido a la alteración en su salud; requiere de un tiempo para la recuperación y, una vez restablecida la capacidad laboral, se produce el retorno al trabajo (Blanca-Gutiérrez et al., 2013; Villaplana, 2014; Álvarez, 2017; Arias et al., 2018; Castellanos, 2019).

En general, como lo reconoce la Organización Internacional del Trabajo en sus postulados, la incapacidad temporal es una condición que solo se atribuye a quienes están en una relación laboral formal; hace parte de los derechos laborales y de la seguridad social (Medina y Morales, 2013; Vásquez, 2013; Mazo y Barrera, 2016; Blasco, 2017), y se otorga por enfermedad y accidente, como lo mencionan Escobar-Aramburo et al. (2013), según la legislación expedida en cada país. Dada su naturaleza y consecuencias, es objeto de estudio de la salud pública (Escobar-Aramburo et al., 2013; González-Ramírez et al., 2017).
Este fenómeno afecta el comportamiento económico y social de un país por sus efectos en la productividad y en el contexto social y familiar (Mekonnen et al., 2018; Carrillo et al., 2019; López et al., 2020). Los estudios sobre el tema han analizado principalmente la relación entre la incapacidad temporal y los efectos en la salud, los costos por baja productividad y la discapacidad (Manent et al., 2016; Álvarez, 2017; López et al., 2020); también, han analizado la asociación entre la incapacidad temporal y aspectos políticos, legales y económicos (Guerrero-López et al., 2012; Mac Master y Echavarría, 2017; Benavides et al., 2018; Kottwitz et al., 2018; Vicente, 2018).

Como se ha indicado, la incapacidad temporal es la afectación temporal de la capacidad laboral de un trabajador o trabajadora, resultante de la afectación en su salud (Valero-Pacheco, 2020). Por esta razón existe la imposibilidad para desempeñar sus roles y actividades habituales, como lo es el trabajo. Implica un tiempo para su recuperación y la garantía de que sus ingresos no se vean afectados mientras retorna al trabajo. En este sentido, los sistemas de seguridad social contemplan el derecho a recibir asistencia y un be- 
neficio económico sustitutivo del salario, lo que en tal contexto se conoce como incapacidad temporal (Ley 776/2002; Restrepo, 2012; Castro, 2013; Vicente, 2014; Yıldız et al., 2015; Sánchez, 2016; Strömberg et al., 2017; Ayala, 2018; Kottwitz et al., 2018; Valero-Pacheco, 2020).

En Colombia existen pocos estudios sobre la incapacidad temporal, particularmente en lo que concierne a la noción desde el ámbito de las normas legales, de la gestión de la salud pública y de los riesgos laborales. En consecuencia, este artículo tiene como propósito examinar la noción de incapacidad temporal vigente en un conjunto de países seleccionados para el estudio y, de esta forma, analizar los elementos que le otorgan calidad y significado al concepto en el contexto de la seguridad social. Los resultados de esta revisión serán el insumo para la reflexión teórica y el estudio de esta figura en el marco del proyecto de investigación Las Incapacidades Temporales como Factor de Ineficiencia y Desperdicio en el Sistema de Salud Colombiano. Fase 1.

\section{Metodología}

Para lograr el objetivo se realizó un estudio cualitativo descriptivo (Vera, 2009). Se aplicó la técnica comparativa (Mancera, 2008) y el análisis crítico (Garcés y Duque, 2007) para el estudio de las expresiones que definen la incapacidad temporal.

Se realizó una búsqueda de literatura relacionada con el tema en las siguientes bases de datos disciplinares e interdisciplinares: Scopus, National Center for Biotechnology Information (NCBI), Science Direct, Scielo, Dialnet, Redalyc y Legis, así como en repositorios de universidades y en sitios web de entidades oficiales. Parte de los documentos incluidos en la revisión fueron publicados entre el 2011-2015, lo cual muestra un importante desarrollo del tema durante ese periodo. Como parte de la estrategia, se extendió la búsqueda hasta el 2020 para ampliar los hallazgos.

Las palabras clave, tomadas de los Descriptores en Ciencias de la Salud (DeCS), que se usaron para la indagación fueron las siguientes: en español, ausencia por enfermedad, incapacidad laboral transitoria, incapacidad temporal, beneficios del seguro, seguridad social, absentismo; en inglés, sick leave, insurance benefits, social security, absenteeism.

Los países seleccionados fueron CoIombia, Argentina, Chile, Perú, Ecuador, México, España y Portugal. Esto porque, bajo el criterio de las y los autores, se trata de países con sistemas de seguridad social comparables con el de Colombia y proveen condiciones de facilidad de acceso y disponibilidad de la información en el periodo indicado.

Los criterios de inclusión fueron: trabajos originales, artículos de revisión e investigación, trabajos o tesis de grado; publicaciones de acceso abierto y texto completo; inclusión de la incapacidad temporal y su relación con salud, seguridad social, derecho a la salud, derecho al trabajo y seguridad y salud en el trabajo; enunciación explícita del concepto o noción de incapacidad temporal y la referencia a alguna de sus condiciones como beneficio de la seguridad social; textos en español e inglés, por ser idiomas dominados por las y los autores. De igual forma, se incluyeron documentos oficiales emitidos por enti- 
dades gubernamentales y normatividad legal de los países de referencia.

La elección inicial de documentos para el estudio fue de 180, de los cuales se eliminaron 128 por no cumplir con los criterios enunciados, de manera que quedaron 52 (figura 1). La revisión fue realizada por cuatro de las y los autores, quienes tienen formación en diferentes disciplinas. A su vez, los artículos fueron evaluados mediante el análisis crítico de forma independiente por cada integrante del grupo. En caso de debate en la elección, se resolvió mediante la discusión grupal.
Se diseñaron matrices para la consolidación manual de las publicaciones, estas incluyeron variables como: año de publicación, nombre de la publicación, resumen, país de origen, idioma, palabras clave, método, resultados y conclusiones o discusión. En el caso de los documentos legales, se elaboró un cuadro descriptivo con identificadores normativos. Por último, se realizó la síntesis de los hallazgos en tres categorías de análisis: elementos conceptuales del término incapacidad temporal, eventos o contingencias objeto de protección y sujetos con derecho a la prestación.

Figura 1. Proceso para la selección de artículos incluidos en la revisión

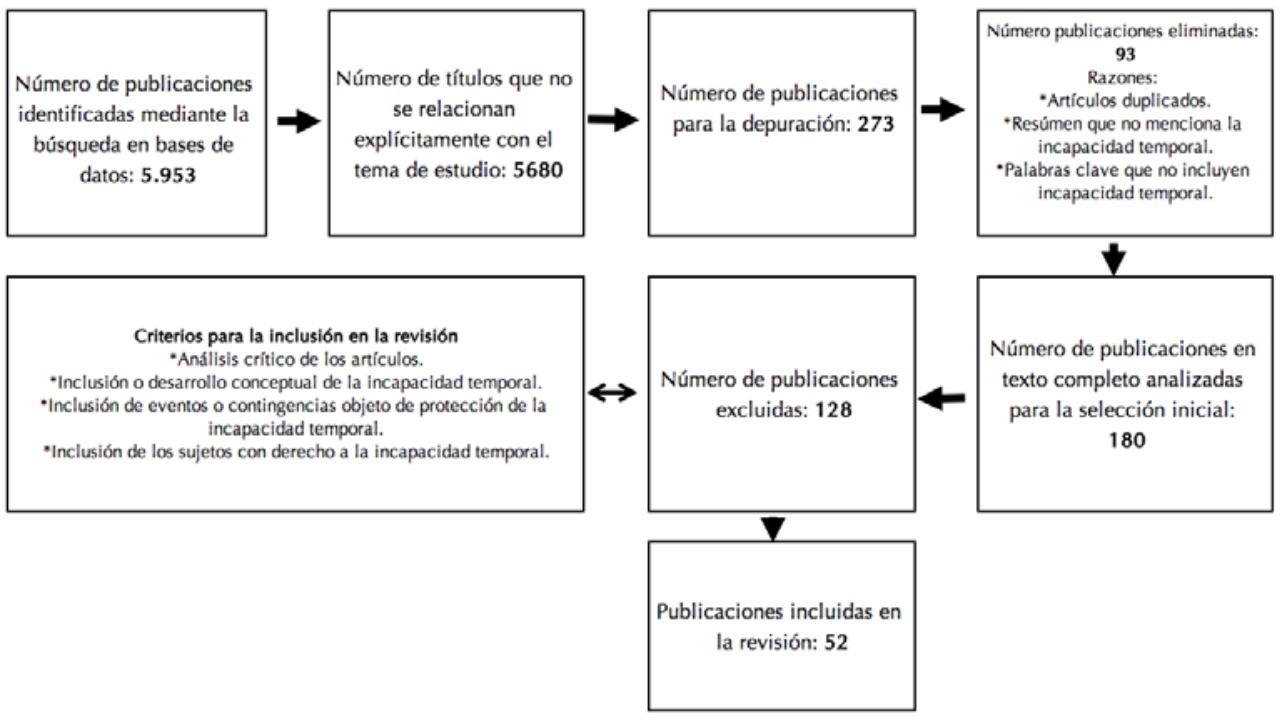

Fuente: elaboración propia. 


\section{Resultados}

A continuación se presentan los resultados del análisis por países en las tres categorías previamente enunciadas: elementos conceptuales del término incapacidad temporal; eventos o contingencias objeto de protección, y sujetos objeto del derecho a la prestación. En la discusión se amplía la comparación entre ellos.

\section{Colombia}

La incapacidad temporal es un derecho de las y los trabajadores. Como beneficio económico se encuentra estipulada en el Sistema General de Salud y en el Sistema General de Riesgos Laborales, componentes del Sistema de Seguridad Social Integral Colombiano (Ley 100/1993; Ley 776/2002). Los descriptores del concepto incluyen el carácter del beneficio, el propósito, la duración y la entidad responsable de garantizar el derecho.

En efecto, es un subsidio que sustituye el salario y su fin es cubrir las necesidades de las y los trabajadores protegiéndoles de los efectos que menoscaban su salud y capacidad económica por enfermedades y lesiones de origen común o laboral (Sentencia T-333/ 2013; Martin-Fumadó, et al., 2014). En Colombia se reconoce el derecho a la incapacidad temporal solo a trabajadoras y trabajadores afiliados tanto al sistema de salud como a riesgos laborales.

En consecuencia, la incapacidad temporal por eventos de naturaleza común será reconocida por las entidades promotoras de salud entre 3 y 180 días y después del día 540. A partir del día 182 y hasta el día 540 es obligación del fondo de pensiones, mientras que la o el empleador asume los dos primeros días (Medina y Morales, 2013; Gómez y Turizo, 2016; Sentencia T-401/2017; Dávila, 2017; Duque et al., 2017; Decreto 1333/2018).

Las contingencias de origen laboral, por su parte, son atendidas por las administradoras de riesgos laborales (Ley 776/2002, art. 1; Gómez y Turizo, 2016; Dávila, 2017; Duque et al., 2017). La prestación se reconoce desde el segundo día hasta la recuperación o la declaración de incapacidad permanente parcial, invalidez o muerte (Ley 776/2002, art. 3; Duque et al., 2017).

\section{Argentina}

En este caso la noción incluye como elementos la situación del trabajador o trabajadora, los efectos en la salud y los eventos o contingencias que le dan origen. Entonces, la incapacidad laboral temporaria, como se le denomina en Argentina, implica el daño (en la salud) sufrido por la o el trabajador y la imposibilidad temporal para ejecutar sus tareas habituales por causa de enfermedad o accidente (común o laboral), siendo una causal para la suspensión del contrato laboral (Ley 20744/1976, art. 208). Esta incapacidad termina cuando se da el alta médica, por declaración de incapacidad laboral permanente, por el transcurso de un año desde la primera manifestación invalidante o por la muerte de la persona damnificada (Ley 24557/1995).

En Argentina, solo los trabajadores y trabajadoras con alguna de las modalidades de contrato reconocidas en su sistema normativo laboral tienen este beneficio (Ley 20744/1976). A su vez, existe diferencia entre los beneficios que se tienen asociados a la incapaci- 
dad temporal cuando la persona trabajadora tiene familia a cargo y por antigüedad (Ley 20744/1976, art. 208).

Argentina reconoce como causas de la prestación las enfermedades y accidentes tanto comunes como laborales y gestiona de forma independiente los riesgos. Es así como, para accidente laboral o enfermedad profesional, la incapacidad laboral temporaria se otorga como una prestación económica hasta que termine el periodo de recuperación, reconociendo la totalidad del ingreso base mensual (Ley 20744/1976, art. 1113; Ley 24557/1995).

\section{Chile}

La incapacidad laboral se define como un derecho de tipo subsidio que permite que el trabajador o trabajadora pueda ausentarse de forma completa o reducir su jornada laboral, según la gravedad de la lesión o la enfermedad, manteniendo en todo momento su remuneración (Contreras, 2016) y hasta su reintegro, como lo estipula la Ley 16744/1968.

En este caso, los trabajadores y trabajadoras dependientes del sector público y privado, así como las y los independientes tienen derecho a la prestación siempre y cuando se encuentren afiliados a un sistema previsional. En el caso de trabajadores y trabajadoras independientes, según la Ley 20.255/2008, deben cumplir con los requisitos de meses de cotización al momento de iniciar la incapacidad (Soto et al., 2015). Como se menciona, son aquellas personas con contrato laboral o independientes quienes ostentan el derecho a la incapacidad temporal; sin embargo, el sistema chileno cuenta con sistemas independientes para la cobertura de contingencias comunes y laborales, según el Decreto con Fuerza de Ley 44/1978.

\section{Perú}

Al igual que los otros países, en Perú la incapacidad temporal es una prestación económica temporal e implica la suspensión del contrato laboral, siendo un derecho exclusivo de las y los trabajadores. Además, debe existir una afectación en la salud que impida la realización de las actividades habituales, como lo indica la Ley 27056/1999.

Como en los casos anteriores, es consecuencia de una enfermedad o accidente sin importar el origen que impida temporalmente realizar las actividades habituales, siendo responsable el Estado tanto del reconocimiento como del pago (Rodríguez y Rodríguez, 2018). Adicionalmente, es posible otorgar incapacidad temporal cuando el trabajo puede traer consecuencias negativas para la recuperación (Rodríguez y Rodríguez, 2018). Para otorgar la prestación es necesario cumplir con los requisitos, entre los cuales está el contar con un contrato laboral vigente, y su reconocimiento varía según el origen de los eventos (común o laboral) (Benavides et al., 2018; Guzmán, 2018).

\section{Ecuador}

Este país contempla la prestación económica de incapacidad temporal como un subsidio cuando existe una inhabilidad temporal de ejercer la labor, necesitando la persona asistencia médica, quirúrgica, hospitalaria o rehabilitación a cargo del Instituto de Seguridad Social (Baldeón, 2014; Resolución C.D. 513/2016). Nuevamente las contingen- 
cias amparadas son enfermedad y accidente, tanto de origen laboral como común, y para su reclamación se requiere el cumplimiento de periodos mínimos de cotización (Baldeón, 2014; Resolución C.D. 513/2016).

Según las normas establecidas en la Resolución C.D. 513/2016, quienes tienen derecho a la incapacidad temporal son todas las personas funcionarias, servidoras públicas y privadas y afiliadas al Seguro General de Riesgos del Trabajo; estas reciben los servicios de prevención, reparación, rehabilitación y reinserción laboral.

\section{México}

El Instituto Mexicano del Seguro Social incluye como elementos de la definición de incapacidad temporal la pérdida de facultades o aptitudes físicas o mentales que limitan parcial o totalmente a la persona asegurada para desempeñar su actividad laboral habitual por un tiempo determinado. La población que accede a la incapacidad temporal son las y los trabajadores permanentes, trabajadores/ trabajadoras eventuales y trabajador/trabajadora eventual del campo (Ley Federal del Trabajo/1970; Ley del Seguro Social/1995; Guerrero-López et al., 2012; Gómez, 2017; Lanz et al., 2020).

El reconocimiento de esta prestación depende del origen del evento (común o laboral), pero, si es por accidente laboral, tiene como efecto el pago de una indemnización a cargo de la o el empleador (Ley Federal del Trabajo/1970).

\section{Portugal}

Tiene un planteamiento similar a los otros países, porque debe existir una le- sión o enfermedad para otorgar la incapacidad, que además impida a la persona trabajar, ya sea por causas laborales o de origen común. Para los casos en los cuales la incapacidad es generada por enfermedad general se le conoce como subsidio por enfermedad, mientras que cuando se deriva de un evento laboral se le Ilama incapacidad temporal por contingencia profesional (Gobierno de España, Ministerio de Trabajo, Migraciones y Seguridad Social, 2019).

En este sentido, la incapacidad temporal se reconoce como una prestación económica a trabajadoras y trabajadores afiliados a la seguridad social, pero la persona debe cumplir ciertos requisitos enunciados en la normatividad legal. En ambos casos, derivada de enfermedad general o evento laboral, su duración es limitada (Gobierno de España, Ministerio de Trabajo, Migraciones y Seguridad Social, 2019).

\section{España}

Coincide con el resto de los países mencionados en que se trata de la situación en la que se encuentran las trabajadoras y los trabajadores impedidos temporalmente para el trabajo y por la cual necesitan asistencia médica, ya sea por efecto de una enfermedad o accidente, sin importar su origen (Gómez, 2018).

La normatividad española reconoce la incapacidad temporal por un tiempo limitado, acompañada de los servicios asistenciales requeridos para la recuperación de la salud. Serán las y los trabajadores con afiliación a la Seguridad Social quienes tengan derecho, si cumplen con los requisitos establecidos (Díaz, 2014; Dávila, 2017; Monereo e Iniesta, 2018; Vicente-Herrero et al., 2018). 


\section{Discusión}

Los elementos comunes en la noción de incapacidad temporal hacen referencia a un mecanismo de protección incluido en el sistema de seguridad social. En síntesis, es una garantía laboral para el trabajador o trabajadora, derivada de la relación de trabajo y aplicable solo a personas vinculadas a una organización o a trabajadores/trabajadoras independientes (Ayala, 2018; Valero-Pacheco, 2020).

En consecuencia, según los artículos y documentos analizados, al otorgar una incapacidad temporal se reconocen prestaciones asistenciales y económicas mientras esta perdure, aunque se contemplan limitaciones en su duración (Ley 20744/1976; Guerrero-López et al., 2012; Martin-Fumadó et al., 2014; Soto et al., 2015; Resolución C.D. 513/2016; Ayala, 2018; Gómez, 2018; Rodríguez y Rodríguez, 2018; Gobierno de España, Ministerio de Trabajo, Migraciones y Seguridad Social, 2019).

Estudiar la incapacidad temporal es importante al ser un problema de salud que justifica la ausencia de la o el trabajador (Escobar-Aramburo et al., 2013) e incluye la percepción subjetiva de la salud, además de factores laborales y extralaborales (Albertí et al., 2012). Desde otro enfoque, es un indicador del estado de salud por su asociación con factores organizacionales, condiciones de trabajo y la duración de la incapacidad temporal (Mazo y Barrera, 2016).

Por lo anterior, analizar los factores que determinan la incapacidad temporal es relevante para la gestión de los servicios de salud (Benavides et al., 2017).
En consecuencia, los marcos legislativos y políticos han evolucionado con el propósito de mejorar las condiciones de aquellos trabajadores y trabajadoras que no puedan realizar su trabajo habitual por razones de salud (Gorelli, 2017). A su vez, la o el empleador debe incluir estrategias encaminadas a disminuir el absentismo laboral, mejorar las condiciones de salud en las y los trabajadores y aumentar la productividad como parte de sus procesos estratégicos (Sánchez, 2015; Benavides et al., 2017; Gorelli, 2017; Mekonnen et al., 2018; López et al., 2020).

Existe un consenso general que determina el principal criterio para otorgar el derecho. Se trata de la presencia del daño en la salud, sin importar el evento o su origen, y que como consecuencia, la persona requiere atención en salud (Ballesteros, 2016) y tiempo para la recuperación; por lo tanto, una vez superado, debe reintegrarse a sus labores habituales (Pardo, 2016; Duque et al., 2017; López et al., 2020).

Las principales diferencias están en la variación de la cotización, los requisitos para el otorgamiento de la incapacidad temporal y la duración de la prestación. En los documentos de los países estudiados se puede evidenciar la existencia de un sistema de seguridad social en el que se incluyen diferentes categorías de trabajadores, eventos y contingencias protegidas, sin embargo, en todos los casos se trata de una prestación finita y que, en caso de no resolverse, evoluciona a otros tipos de prestación como la indemnización o pensión por invalidez (Ballesteros, 2016). 


\section{Conclusiones}

Los resultados demuestran que, en los países analizados, los conceptos coinciden en los aspectos fundamentales para el otorgamiento, la duración y el pago de la incapacidad temporal, como una garantía de la seguridad social destinada a trabajadores y trabajadoras formales. Lo anterior deja claro que, aun tratándose de sistemas de seguridad social particulares para cada país, la incapacidad temporal es una prestación universal y finita enmarcada en el contexto de los derechos laborales, de la salud y de la seguridad social.

En este contexto, la incapacidad temporal es un factor de estudio desde la gestión de los servicios de salud y riesgos laborales por estar relacionada con las variables determinantes de la salud de la población trabajadora, además de considerarse como un primer acto de determinación de la pérdida de capacidad laboral que, aunque temporal, puede desencadenar en el retiro temprano de la fuerza laboral.

Es importante que las contribuciones de estudios en esta materia permitan conocer el estado de la incapacidad temporal en Colombia desde una perspectiva interdisciplinar, de tal forma que favorezcan el diseño de estrategias aplicables en los ámbitos nacional, regional y local con enfoque de equidad y considerando la ocupación y el ámbito laboral. Por otra parte, faltan estudios que propongan intervenciones para el reintegro laboral y el manejo de la discapacidad en el trabajo, lo que puede aumentar el absentismo laboral con altos costos laborales y sociales.
Una limitación del estudio está en la revisión de información de algunos países, sin embargo, desde la posición del grupo de autores y autoras, estos cuentan con sistemas de seguridad social comparables con el de Colombia. Otra posible limitación es la elección de los idiomas para la búsqueda de información, pues la revisión en portugués, por ejemplo, podría haber aumentado los hallazgos.

Financiamiento: el proyecto de investigación del cual se deriva este artículo es financiado por la Universidad de Bogotá Jorge Tadeo Lozano, mediante Convocatoria Interna de Investigación n. ${ }^{\circ} 17$ de 2018 de la Dirección de Investigación, Creatividad e Innovación. 


\section{Referencias}

Albertí, C., Jardí, J., Manzanera, R., Torá, I., Delclós, J. y Benavides, F. (2012). Incapacidad temporal por enfermedad común y accidente no laboral en Cataluña, 20072010. Archivos de Prevención de Riesgos Laborales, 15(4), 172-177. https://dx.doi. org/10.12961/aprl.2012.15.4.02

Álvarez, J. (2017). Alteración de la salud, asistencia sanitaria y control médico de las situaciones de incapacidad temporal para el trabajo. Revista de Derecho de la Seguridad Social, Laborum, (12), 87-104. http:// revista.laborum.es/index.php/revsegsoc/ article/view/236

Antonio, M. (2011). Problemática multifactorial del absentismo laboral, el presentismo y la procastinación en las estructuras en que se desenvuelve el trabajador. Medicina y Seguridad del Trabajo, 57(223), 111-120. http://scielo. isciii.es/scielo.php?script=sci abstract\&pid=S0465-546X2011000200004

Arias, D, Rodríguez, A., Zapata, J. y Vásquez, E. (2018). Incapacidad laboral por desórdenes músculo esqueléticos en población trabajadora del área de cultivo en una empresa floricultora en Colombia. Revista de la Asociación Española de Especialistas en Medicina del Trabajo, 27(3), 166-174. http://scielo. isciii.es/scielo.php?script=sci arttext\&pi$\mathrm{d}=\mathrm{S} 1132-62552018000300166 \& \ln -$ $\mathrm{g}=\mathrm{es} \& \operatorname{tnng}=\mathrm{es}$

Ayala, S. (2018). Marco jurídico-conceptual del sistema público de los Servicios Sociales. Barataria. Revista Castellano-Manchega de Ciencias Sociales, (24), 225-234. https://dx.doi.org/10.20932/barataria. $\underline{\mathrm{v} 0 \mathrm{i} 24.398}$
Baldeón, M. (2014). Análisis estadístico de accidentalidad laboral del Ecuador y comparación con la accidentalidad laboral de Colombia del año 2013 [tesis de maestría, Universidad San Francisco de Quito]. Repositorio Digital Universidad San Francisco de Quito. http://repositorio.usfq.edu. ec/handle/23000/3549

Ballesteros, M. (2016). Conflictos bioéticos en la relación sanitaria derivada de las bajas laborales. Revista de Bioética y Derecho, (38), 131-147. https://dx.doi.org/10.1344/ rbd2016.38.17050

Benavides, E., Coronado, J., Mendoza, C., Paz, A. y Tofenio, J. (2018). Diseño de una solución integral para el proceso de subsidio de incapacidad temporal de trabajo utilizando la tecnología Blockchainel [tesis de maestría, Universidad Esan]. Repositorio Institucional Universidad Esan. https://hdl. handle.net/20.500.12640/1396

Benavides, F., Delclós, J. y Serra, C (2018). Estado de bienestar y salud pública: el papel de la salud laboral. Gaceta Sanitaria. 32 (4), 377-380. https://dx.doi.org/10.1016/j. gaceta.2017.07.007

Benavides, F., Zaballa, E., Duran, X., Sanchez-Niubo, A. y Porras, D. (2017). Incidencia de la incapacidad temporal por contingencia común en España según la actividad económica de la empresa. Archivos de Prevención de Riesgos Laborales, 20(1), 14-25. https:// dx.doi.org/10.12961/aprl.2017.20.01.3

Blanca-Gutiérrez, J., Jiménez-Díaz, M. y Escalera-Franco, L. (2013). Intervenciones eficaces para reducir el absentismo del personal de enfermería hospitalario. Gaceta Sanitaria, 27(6), 545-551. http://dx.doi.org/10.1016/j. gaceta.2012.09.006 
Blasco, J. (2017). La convergencia en el derecho a las prestaciones económicas de la seguridad social. Revista de información laboral,(10), 59-101. https://dialnet.unirioja.es/servlet/articulo?codigo $=6174901$

Carrillo, D., Montes, L., Giraldo, J., Méndez, J., Cruz, M., Vásquez, E. y Valencia, N. (2019). Absentismo laboral por incapacidad médica en un centro de contacto de la ciudad de Medellín en el periodo 2016-2017. Revista de la Asociación Española de Especialistas en Medicina del Trabajo, 28(1), 49-56. http://scielo. isciii.es/scielo.php?script=sci arttext\&pi$\mathrm{d}=\mathrm{S} 1132-62552019000100006 \& \ln -$ $\mathrm{g}=\mathrm{es} \& \ln \mathrm{ln}=\mathrm{es}$

Castellanos, J. (2019). Incapacidad como acto médico y ley estatutaria de salud. Universitas Medica, 60(1). https://dx.doi. org/10.11144//averiana.umed60-1.imle

Castro, J. (2013). La calificación integral y la pensión de invalidez con doble origen en el ordenamiento legal colombiano: aportes para su precisión e interpretación jurisprudencial [tesis de maestría, Universidad Nacional de Colombia]. Repositorio Institucional UN. https://repositorio.unal.edu. co/handle/unal/12202

Congreso de la Nación Argentina. (1976, 13 de mayo). Ley 20744 de 1976. Ley de Contrato de Trabajo. https://www.ilo.org/dyn/travail/ docs/979/Ley\%20N\%\%2020.744.pdf

Congreso de la Nación Argentina. (1995, 13 de septiembre). Ley 24557 de 1995. Riesgos del Trabajo. Diario Oficial n. ${ }^{\circ} 28242$. https://www.ilo.org/dyn/travail/docs/1467/ RIESGOS\%20DEL\%20TRABAJO.pdf

Congreso de la República de Colombia (1993, 23 de diciembre). Ley 100 de 1993. Sistema de Seguridad Social Integral. Dia- rio Oficial n. ${ }^{\circ}$ 41148. http://www.secretariasenado.gov.co/senado/basedoc/ ley 0100 1993.html

Congreso de la República de Colombia (2002, 17 de diciembre). Ley 776 de 2002. Por la cual se dictan normas sobre la organización, administración y prestaciones del Sistema General de Riesgos Profesionales. Diario Oficial n. ${ }^{\circ}$ 45037. https://www. alcaldiabogota.gov.co/sisjur/normas/Norma1.jsp?i=16752

Congreso de los Estados Unidos Mexicanos. (1995, 21 de diciembre). Ley del Seguro Social. Diario Oficial del 21 de diciembre de 1995. http://www.diputados.gob.mx/ LeyesBiblio/ref/Iss/LSS orig 21dic95.pdf

Congreso de los Estados Unidos Mexicanos. (1970, 1 de abril). Ley Federal del Trabajo. Diario Oficial n. ${ }^{\circ}$ 26. http://www.diputados.gob.mx/LeyesBiblio/ref/lft/LFT orig $01 \mathrm{abr} 70$ ima.pdf

Congreso Nacional de Chile. (1968, 23 de enero). Ley 16744 de 1968. Establece normas sobre accidentes del trabajo y enfermedades profesionales. Diario Oficial n. ${ }^{\circ}$ 26957. https://www.bcn.cl/leychile/navegar?idNorma $=28650$

Contreras, Y. (2016). Análisis del impacto económico del ausentismo laboral por licencias médicas en el personal de la Universidad del Bio-Bio, sede Chillán [tesis de maestría, Universidad del BioBio]. Repositorio Digital Universidad del Bio Bio. http://repobib.ubiobio.cl/jspui/hand$\underline{\text { le/123456789/1689 }}$

Corte Constitucional, República de Colombia (2013, 11 de junio). Sentencia T-333 de 2013 (Luis Ernesto Vargas Silva M.P.). https://www.corteconstitucional.gov.co/relatoria/2013/T-333-13.htm 
Corte Constitucional, República de Colombia. (2013, 23 de junio). Sentencia T-401 de 2017 (Gloria Stella Ortíz Delgado, M.P.). https://www.corteconstitucional.gov.co/ relatoria/2017/t-401-17.htm

Dávila, J. (2017). Subsidio por incapacidad como prestación económica a cargo del sistema integral de seguridad social. Aproximaciones a las responsabilidades de los distintos subsistemas. Páginas de Seguridad Social. Universidad Externado de Colombia, 1(2), 113-127. https://dx.doi. org/10.18601/25390406.n2.05

Díaz, C. (2014). Reflexiones sobre la incapacidad y aptitud laboral. Nuevas sinergias entre medicina evaluadora y medicina laboral. Historia clínica laboral única. La incapacidad laboral, un continuo evolutivo. Medicina y Seguridad del Trabajo (Suplemento Extraordinario 1), 125-130. http://scielo.isciii.es/pdf/mesetra/v60s1/ ponencia17.pdf

Duque, S., Quintero, M. y González, P. (2017). El pago de incapacidades por enfermedad común y el derecho al mínimo vital de un trabajador en Colombia. Revista Facultad de Derecho y Ciencias Políticas, 47(127), 383-405. http://dx.doi.org/10.18566/rfdcp.v47n127.a05

Escobar-Aramburo, M., Duarte-Suárez, M., Caicedo-Campo, L., García, M., Valderrama-Aguirre, A. y Cruz, A. (2013). Ausentismo laboral por enfermedad de origen infeccioso en una institución forense. Revista Colombiana de Salud Ocupacional, 3(2), 12-17. https://revistas.unilibre.edu. co/index.php/rc_salud_ocupa/article/ view/4859

Garcés, J. y Duque, E. (2007). Metodología para el análisis y la revisión crítica de artículos de investigación. Innovar,
17(29), 184-194. http://www.scielo. org.co/scielo.php?script=sci arttext\&pi$\mathrm{d}=\mathrm{S} 0121-50512007000100011 \& \ln -$ $\mathrm{g}=\mathrm{en} \& \operatorname{lng}=\mathrm{es}$

Gobierno de España, Ministerio de Trabajo, Migraciones y Seguridad Social. (2019). Prestación por incapacidad temporal en Portugal. Revista de Actualidad Internacional Sociolaboral, (238), 181-186. http:// www.mites.gob.es/ficheros/ministerio/ mundo/revista ais/238/Portugal.pdf

Gómez, C. (2017). El Sistema de Salud en México. Revista Conamed, 22(3), 129-135. https://dialnet.unirioja.es/servlet/articulo?codigo $=6434801$

Gómez, M. (2018). Discapacidad, incapacidad temporal duradera y discriminación: ¿Es nulo el despido de un trabajador en situación de incapacidad temporal? Revista de Derecho de la Seguridad Social Laborum (14), 67-88. https://revista.laborum.es/index.php/revsegsoc/article/ view $/ 266$ ? acceptCookies $=1$

Gómez, R. y Turizo, P. (2016). Seguridad y salud en el trabajo en Colombia: retos frente a las personas con discapacidad. Revista CES Derecho. 7(2), 84-94. https://doi. org/10.21615/4075

González-Ramírez, C., Montanero-Fernández, J. y Peral-Pacheco, D. (2017). A multifactorial study on duration of temporary disabilities in Spain. Archives of Environmental \& Occupational Health, 72(6), 328-335. https://dx.doi.org/10.1080/19338244.201 $\underline{6.1246410}$

Gorelli, J. (2017). El problemático control de la incapacidad temporal en el régimen general. Temas Laborales (136), 13-48. https:// dialnet.unirioja.es/servlet/articulo?codigo $=6552118$ 
Guerrero-López, C., Reynales-Shigematsu, L., Jiménez-Ruiz, J., Karam-Araujo, R., Maldonado-Cruz, C. y Camacho-Solís, R. (2012). Costos por ausentismo laboral atribuibles al consumo de tabaco en el Instituto Mexicano del Seguro Social y en México, 2006-2009. Salud Pública de México, 54(3), 233-241. http://www.scielo. org. $\mathrm{mx} /$ scielo.php? script=sci arttext\&pi$\mathrm{d}=$ S0036-36342012000300005

Guzmán, L. (2018). Costos en el Sistema de Prestaciones Económicas de EsSalud: subsidios a cargo del empleador. Revista Derecho \& Sociedad (50), 115-123. http:// revistas.pucp.edu.pe/index.php/derechoysociedad/article/view/20377

Instituto Ecuatoriano de Seguridad Social. (2016, 4 de marzo). Resolución C.D. 513 de 2016. Reglamento del Seguro General de Riesgos del Trabajo. Registro Oficial n. ${ }^{\circ}$ 632. https://sut.trabajo.gob.ec/publico/ Normativa\%20Legal/Resoluciones/Resolución\%20del\%20IESS\%20513.pdf

Kottwitz, M., Schade, V., Burger, C., Radlinger, L. y Elfering, A. (2018). Time pressure, time autonomy, and sickness absenteeism in hospital employees: a longitudinal study on organizational absenteeism records. Safety and Health at Work, 9(1), 109-114. https://dx.doi.org/10.1016/j. shaw.2017.06.013

Lanz, J., Haro, M., Quiñones, K., Rivera, D. y Ayala, G. (2020). Retro-información a médicos familiares para optimizar la prescripción de certificados de incapacidad temporal en una unidad médico familiar. Revista Cubana de Salud y Trabajo, 19(3), 03-15. http://www.revsaludtrabajo.sld.cu/ index.php/revsyt/article/view/159
López, C., Bogotá, L., Valero-Pacheco, I., Torres, C. y Castillo, A. (2020). La incapacidad temporal y variables relacionadas: revisión bibliográfica. Ciencia y Tecnología para la Salud Visual y Ocular, 17(2), 21-31. https://dx.doi.org/10.19052/sv.vol17.iss2.3

Ministerio del Trabajo y Previsión Social. (1978, 1 de junio). Decreto 44 de 1978 [con fuerza de ley]. Fija normas comunes para los subsidios por incapacidad laboral de los trabajadores dependientes del sector privado. Diario Oficial n. 30123. https://www.bcn.cl/leychile/navegar?id$\underline{\text { Norma }=4252}$

Ministerio de Trabajo y Previsión Social. (2008, 11 de marzo). Ley 20255 de 2008. Establece reforma previsional. Diario Oficial n. ${ }^{\circ}$ 39014. https://www.bcn.cl/leychile/navegar?idNorma=269892

Mac Master, B. y Echavarría, A. (eds.) (2017). Salud y estabilidad en el empleo: retos jurídicos y económicos para la sostenibilidad de las empresas (1a ed.). Asociación Nacional de Empresarios de Colombia (ANDI) - Editorial Mundo Libro.

Mancera, A. (2008). Consideraciones durante el proceso comparativo. Boletín Mexicano de Derecho Comparado, 41(121), 213-243. http://www.scielo. org. $\mathrm{mx} /$ scielo.php?script=sci arttext\&pid $=$ S0041-86332008000100007\&l$\underline{\text { ng}}=\mathrm{es} \& \ln g=\mathrm{es}$

Manent, I., Ramada, J. y Serra, C. (2016). Duración y características de los episodios de incapacidad temporal por trastornos músculo-esqueléticos en Cataluña, 20072010. Archivos de Prevención de Riesgos Laborales, 19(4), 222-230. https://dx.doi. org/10.12961/aprl.2016.19.04.3 
Martin-Fumadó, C., Martí, G., Puig, Ll. y Arimany-Manso, J. (2014). La incapacidad temporal y sus implicaciones legales. Medicina Clínica, 142 (Supplement 2), 37-42. https://dx.doi.org/10.1016/S00257753(14)70070-3

Mazo, D. y Barrera, L. (2016). Factores relacionados con el absentismo laboral por causa médica en el personal de enfermería, Fundación Clínica del Norte, 2013 - 2014. CES Salud Pública, 7(1), 3-16. https:// revistas.ces.edu.co/index.php/ces salud publica/article/view/3280/2640

Medina, L. y Morales, C. (2013). Análisis del ausentismo laboral por incapacidad temporal de una universidad pública del suroccidente colombiano en el periodo 2005 a 2009 [trabajo de maestría, Universidad del Valle]. Repositorio Digital Universidad del Valle. http://hdl.handle. net/10893/10519

Mekonnen, T., Tefera, M. y Melsew, Y. (2018). Sick at work: prevalence and determinants among healthcare workers, western Ethiopia: an institution based cross-sectional study. Annals of Occupational and Environmental Medicine, 30(2), 1-10. https:// dx.doi.org/10.1186/s40557-018-0213-4

Ministerio de Salud y Protección Social. (2018, 27 de julio). Decreto 1333 de 2018. Por el cual se sustituye el Título 3 de la Parte 2 del Libro 2 del Decreto 780 de 2016, se reglamenta las incapacidades superiores a 540 días y se dictan otras disposiciones. Diario Oficial n. ${ }^{\circ}$ 50667. https://dapre. presidencia.gov.co/normativa/normativa/ DECRETO \%201333\%20DEL \%2027\%20 DE\%20JULIO\%20DE\%202018.pdf

Monereo, J. e Iniesta, G. (2018). Las incapacidades laborales en el punto de mira de la administración de la seguridad social entre exigencias "control" y necesidad ineludible de "racionalización integral" de su tratamiento jurídico. Revista de Derecho de la Seguridad social. Laborum, (17), 23-30. https://dialnet.unirioja. es/servlet/articulo?codigo $=6807837$

Pardo, V. (2016). Hombro doloroso e incapacidad temporal. El retorno al trabajo tras larga baja por hombro doloroso. Causalidad del trabajo en el hombro doloroso. Medicina y Seguridad del Trabajo, 62 (245), 337-359. http://scielo.isciii.es/pdf/ mesetra/v62n245/06 inspeccion.pdf

Presidencia de la República del Perú. (1999, 29 de enero). Ley 27056 de 1999. Ley de creación del Seguro Social de Salud (ESSALUD). Diario Oficial n. ${ }^{\circ}$ 6794. http:// www.essalud.gob.pe/transparencia/pdf/ Decreto supremo 00299 TR.pdf

Restrepo, C. (2012). Entorno regulatorio y ausentismo laboral. Una aproximación teórica. Sotavento M.B.A(19), 52-62. https://revistas.uexternado.edu.co/index. php/sotavento/article/view/3340

Rodríguez, C. y Rodríguez, S. (2018). Subsidio por incapacidad temporal no tramitado del personal de ESSALUD y su repercusión económica en la red asistencial Lambayeque, Julio-Diciembre 2016 [tesis de maestría, Universidad César VaIlejo]. Respositorio Digital Institucional Universidad César Vallejo. https://hdl. handle.net/20.500.12692/21793

Sánchez, D. (2015). Ausentismo laboral: una visión desde la gestión de la seguridad y la salud en el trabajo. Revista Salud Bosque, 5(1), 43-53. https://dx.doi. org/10.18270/rsb.v5i1.182 
Sánchez, L. (2016). Datos epidemiológicos en incapacidad temporal en el Instituto Nacional de la Seguridad Social. Medicina y Seguridad del Trabajo, 62(Supl. Extra), 3134. http://scielo.isciii.es/scielo.php?script=sci arttext\&pid=S0465-546X2016000400003\&lng=es\&tlng=es

Soto, A., Herrera, R. y Fuentes, R. (2015). Efecto de la seguridad social en la duración del ausentismo laboral en el Servicio de Salud de Ñuble: un análisis de supervivencia. Revista Médica de Chile, 148(8), 987994. http://dx.doi.org/10.4067/S0034$\underline{98872015000800004}$

Strömberg , C., Aboagye, E., Hagberg , J., Bergström, G. y Lohela-Karlsson, M. (2017). Estimating the effect and economic impact of absenteeism, presenteeism, and work environment-related problems on reductions in productivity from a managerial perspective. Value Health, 20(8), 1058-1064. http://dx.doi.org/10.1016/j. jval.2017.05.008

Valero-Pacheco, I. (2020). La incapacidad temporal y la enfermedad relacionada con el trabajo en la seguridad social. Una aproximación a partir de la revisión de literatura. Verba luris (44), 71-99. https:// dx.doi.org/10.18041/0121-3474/verbaiuris. 44.6869

Vásquez, E. (2013). Absentismo laboral por causa médica en trabajadores del área operativa de una compañía de extracción de minerales en Colombia, 2011. Medicina y Seguridad del Trabajo, 59(230), 93-101. https://dx.doi.org/10.4321/S0465546X2013000100006

Vera, O. (2009). Cómo escribir artículos de revisión. Revista Médica La Paz, 15(1), 63-69. http://www.scielo.org. bo/scielo.php?script=sci arttext\&pi-
$\underline{d}=\mathrm{S} 1726-89582009000100010 \& \ln -$ $\mathrm{g}=\mathrm{es} \& \ln \mathrm{ln}=\mathrm{es}$

Vicente, J. (2014). La valoración de la profesión, del trabajo, de la ocupación, y de las tareas en el en España. Actualización legislativa 2016. Revista Médica del Instituto Mexicano del Seguro Social, 56(1), 84-91. https://www.medigraphic.com/ pdfs/imss/im-2018/im181m.pdf

Villaplana, M. (2014). Análisis de la influencia de los factores relacionados con los indicadores de la Incapacidad Temporal y la reincorporación al trabajo. Medicina y Seguridad del Trabajo, 60(Supl. 1), 65-73. http:// scielo.isciii.es/scielo.php?script=sci arttext\&pid=S0465-546X2014000500012

Yıldız, H., Yıldız, B., Zehira, C. y Aykaç, M. (2015). The antecedents of presenteeism and sickness procedimiento de valoración de la capacidad laboral en materia de seguridad social. Medicina y Seguridad del Trabajo, 60(237), 660674. https://dx.doi.org/10.4321/S0465546X2014000400006

Vicente, J. (2018). La gestión de la incapacidad laboral algo más que una cuestión económica. Medicina y Seguridad del Trabajo, 64(251), 131-160. http://scielo. isciii.es/scielo.php?script=sci arttext\&pi$\underline{d}=$ S0465-546X2018000200131\&lng=es

Vicente-Herrero, M., Terradillos-García, M., Capdevila-García, L., Ramírez-Íñiguez de la Torre, M., Aguilar-Jiménez, E., Aguado-Benedí, M., López-González, A. y Torres-Alberich, J. (2018). Discapacidad e incapacidad laboral absenteeism: A research in turkish health sector. Procedia - Social and Behavioral Sciences, 207(20), 398-403. https://dx.doi.org/10.1016/j.sbspro.2015.10.109 